\title{
A classroom deployment of a haptic system for learning cell biology
}

Conference or Workshop Item

Accepted Version

Tokatli, O., Tracey, M., Hwang, F., Barrett, N., Jones, C., Johnson, R., Webb, M. and Harwin, W. (2018) A classroom deployment of a haptic system for learning cell biology. In: Eurohaptics 2018, 13-16 Jun 2018, Pisa, Italy. (Published in Lecture Notes in Computer Science, volumes 10893 and 10894) Available at http://centaur.reading.ac.uk/76658/

It is advisable to refer to the publisher's version if you intend to cite from the work. See Guidance on citing.

Published version at: https://eurohaptics2018.org/

All outputs in CentAUR are protected by Intellectual Property Rights law, including copyright law. Copyright and IPR is retained by the creators or other copyright holders. Terms and conditions for use of this material are defined in the End User Agreement. 


\section{www.reading.ac.uk/centaur}

\section{CentAUR}

Central Archive at the University of Reading

Reading's research outputs online 


\title{
A Classroom Deployment of a Haptic System for Learning Cell Biology
}

\author{
Ozan Tokatli $^{\dagger}$, Megan Tracey ${ }^{\ddagger}$, Faustina Hwang ${ }^{\dagger}$, Natasha Barrett ${ }^{\star}$, Chris \\ Jones $^{\star}$, Ros Johnson ${ }^{\circ}$, Mary Webb ${ }^{\ddagger}$, William Harwin ${ }^{\dagger}$ \\ ${ }^{\dagger}$ Biomedical Engineering, School of Biological Sciences, University of Reading \\ $¥$ School of Education, Communication \& Society, King’s College London \\ * Biomedical Sciences, School of Biological Sciences, University of Reading \\ $\circ$ The Abbey School
}

\begin{abstract}
The use of haptic systems in the classroom for enhancing science education is an underexplored area. In the education literature, it has been reported that certain concepts in science education are difficult for students to grasp and, as a result, misconceptions can be formed in the students' knowledge. We conducted a study with 62 Year 8 (typically 12-13 years old) students who used a haptic application to study cell biology, specifically the concept of diffusion across a cell membrane. The preliminary analysis of the feedback from the students suggests opportunities for haptic applications to enhance their learning, and also highlights a number of points to consider in the design of the application, including the choice of haptic interface and the design of the virtual environment.
\end{abstract}

Keywords: Haptic and Education, Haptic Diffusion Simulation, Haptic Rendering

\section{Introduction}

Education and skills training provide a key application area for haptic technology, yet provides challenge that range from control and stability, through to haptic rendering and pedagogy. Medical, veterinary and dental skills training forms the core of much research in this domain $[14,20]$. Recently authors have begun to explore the use of haptic devices in engineering education at university level $[18,15,9,5,16]$. A third group that may benefit from haptic principles in education are students in secondary education (that is in the age range of approximately 11 to 18 years old). Scientific concepts are sometimes particularly difficult to grasp and a haptic approach for this constituency may afford students a chance to use a 'hands-on' approach to learn and test principles across a range of subjects.

\section{Background}

Technology enhanced learning (TEL) is a general term that is applied widely in secondary school education. A wide range of technologies from 
mobile computing to virtual reality installations are considered within TEL. However educational content has largely been provided as a two dimensional representation, and interaction when provided to the students has been either touch screen gestures, or mouse and keyboard interactions. But there is a growing interest in visualising 3-D structures, with most work centred on biology ranging from cell structures to the cardiovascular system $[11,3,10]$. Haptic interfaces can offer a natural way to interact and explore this rich 3-D learning environment since it encourages visual and tactile (haptic) interactions and may facilitate student cooperation as well as providing students with opportunities to hypothesise and explore complex scientific concepts.

In science education, hands-on practical work, in which individuals or small groups of students manipulate the objects or materials they are studying, has often been highly-valued by teachers as a pedagogical approach that motivates students and helps to develop their understanding. The benefits of practical work in science education include enhancing the learning of scientific knowledge, challenging students' misconceptions of scientific ideas and processes, teaching laboratory skills, enabling insight into and expertise in scientific method as well as stimulating students' interest and increasing motivation to study science beyond school [13]. In some areas of science a rich multi-sensory learning experience can be achieved using physical objects but many areas involve visualising structures and processes that cannot easily be observed directly, or for which cost or ethical considerations prove prohibitive.

It has long been recognised that the ability to visualise and to manipulate objects in the imagination is a crucial skill for learning science (for example see the review of $3 \mathrm{D}$ visualisation in chemistry [21]) but this is not easily achieved through the $2-\mathrm{D}$ representations and static $3-\mathrm{D}$ models frequently used in science classrooms [23]. Technology enhanced learning (TEL) can support the development of visualisation skills [17], the learning of difficult concepts and enable hypothesis testing in areas of science learning where direct manipulation of real-world objects is not possible $[19,23]$. However TEL in science has mainly consisted of simulations, animations, modelling, measurement and control devices and online learning environments, where the interaction remains largely one of mouse clicks and windows menus, an interface method that is poorlysuited for 3D interactions [8].

Haptic interfaces are synonymous with the haptic sense, that is a sensorymotor interaction, that underlies natural interactions and helps to calibrate visual cues [7]. Haptic technologies give the learner a sensation of kinaesthetic feedback in conjunction with auditory and visual sensory input while the learner is engaged in the cognitive processing necessary to learn a procedure.

\section{Curriculum and content}

The use of visualisation tools in school is a natural consequence of the steady improvement in computers. The Abbey School, Reading introduced 3D stereo projectors into their teaching in 2009 and has since 
used it at specific points in their biology curriculum [10]. The students are given shutter glasses during their lesson and the software allows the teacher to manipulate 3D models of relevant biological simulations via a $3 \mathrm{D}$ mouse, thus allowing students to appreciate the spatial relationships between features.

In a small trial run in 2010, students in their first year at the school (aged 11) were introduced to cell structures either via traditional teaching, or via a lesson that included the chance to use shutter glasses to observe a cell model that the teacher could manipulate. The post test was to construct a cell and the teachers observed a strong correlation between students observing the stereoscopic cell model and their subsequent ability to visualise the cell in three dimensions $[10,11]$.

The complexity, scale and importance of cell biology makes it an interesting domain to introduce better interaction with content via haptic interactions. The problems experienced by students in visualising the three dimensional nature of cells may be a consequence of the difficulty of making direct observation of cell processes. Thus most educational content is in terms of cross sectional schematics, or observations via a light microscope, and although more educational material is now available as interactive websites [2] or high quality animations[4] these still do not allow students to explore on their own terms.

In this paper we are presenting a cell simulation that allows students to observe the particle motion and interact with particles diffusing through the cell membrane. We hypothesise that the proposed haptic learning approach can help to reinforce specific educational concepts and can foster collaborative and active learning.

\section{Experimental Setup}

\subsection{Multi-finger Haptic System}

The experimental setup, shown in Fig. 1, supports a pair of students, designated a pilot and a co-pilot, to work collaboratively. The pilot, who wears a head-mounted display (an Oculus Rift for this study) and is immersed into the virtual world, interacts with the virtual environment using the haptic interface. The co-pilot shares the same view as the pilot but using a computer screen, and uses the keyboard for certain interactions such as application controls.

The haptic interface setup consists of two Phantom robots [12]. The device is controlled by a single hand (either left or right) and the fingers of the user (thumb and index fingers) are attached to the Phantom robots by thimble linkages which allow 3 DoF rotation. The multi-finger haptic interface allows 5 DoF manipulation of objects. The location of each finger in space is calculated using the forward kinematics of the robot. However, there is no orientation sensing for the fingers of the user and so the rotation of virtual objects along the axis connecting the fingers is not possible. The workspace dimensions of the haptic device are $30 \mathrm{~cm}$ along the x-axis, $23 \mathrm{~cm}$ along the y-axis and $40 \mathrm{~cm}$ along the z-axis. When manipulating an object with two fingers, the device allows rotations of $360^{\circ}$ about the $\mathrm{x}$-axis, $180^{\circ}$ about the $\mathrm{y}$-axis and $180^{\circ}$ about the $\mathrm{z}$-axis. 


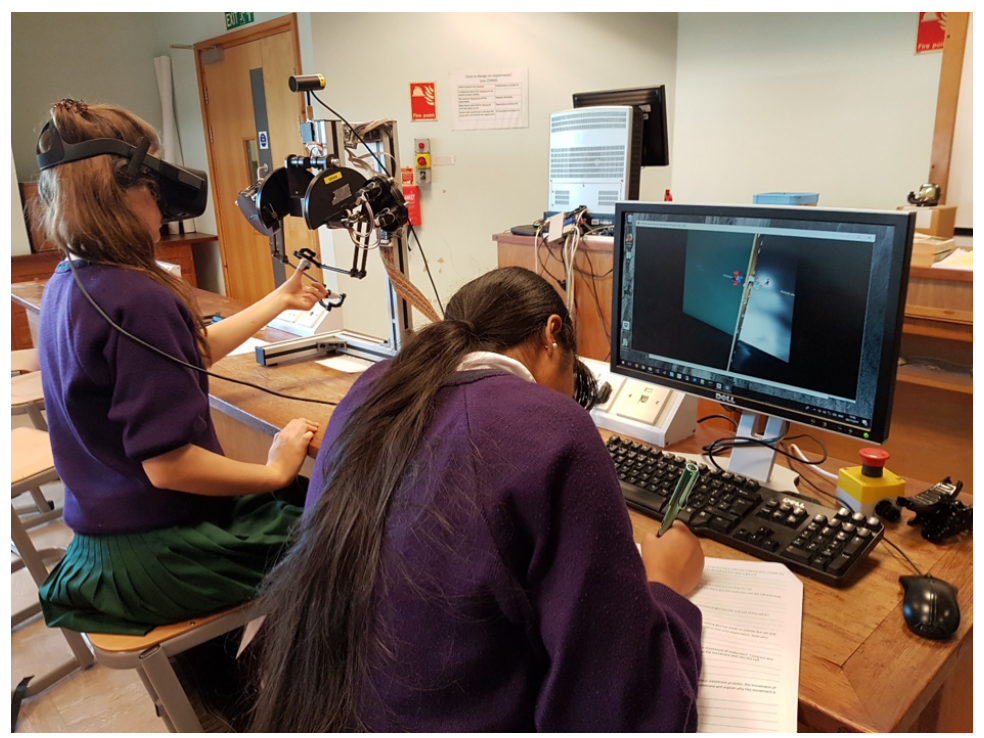

Fig. 1. The multi-finger haptic interface used by the pilot while the co-pilot is working on the worksheet. The device is controlled by a single hand where the fingers of the user (thumb and index fingers) are attached to the robots by a thimble.

One of the mechanical design challenges of the haptic interface is achieving a flexible solution for attaching various sizes of fingers to the robots of the haptic interface. This problem becomes significant as the target group in the experiments is boys and girls of age around 12 who have a wide range of finger and thumb diameters. The thimble design is used in Fig. 1 to attach the student's finger to the haptic interface. This design provides flexibility for a wide range of finger sizes and aims to ensure that the finger is firmly attached while not being uncomfortable to the user.

\subsection{The Virtual Environment}

In this project, we created a virtual environment to simulate diffusion across the cell membrane and a concentration gradient for particles at cellular level (Fig. 2). In this virtual environment, the planar object dividing the world into two halves represents the cell membrane. The texture of this object depicts the lipid bilayer which is the building blocks of the membrane. White objects embedded into the membrane represent membrane proteins and the channels which are used to transport certain molecules/particles from one side of the membrane to the other. These structures are freely floating within the membrane and the student moving them feels a resistive force in response to movement. The interior of the cell (cytoplasm) and the outside of the cell (extracellular space) are labelled to help the students relate the virtual environment to their 
prior cell knowledge. The virtual environment contains various particles (Fig 2 is depicting oxygen $\left(\mathrm{O}_{2}\right)$ and carbon dioxide $\left(\mathrm{CO}_{2}\right)$. Other particles in the environment are sodium $(\mathrm{Na})$, potassium $(\mathrm{K})$ and glucose $\left.\left(\mathrm{C}_{6} \mathrm{H}_{12} \mathrm{O}_{6}\right)\right)$ which are moving under a random force to represent the Brownian motion of the particles. The choice of particle types is based on the biology curriculum of Year 8 students, hence other particles are omitted for the virtual environment. While the cell membrane is transparent to $\mathrm{O}_{2}$ and $\mathrm{CO}_{2}$, i.e. they can pass freely through the membrane, the other particles are not free to pass through the membrane. As a result the particle will bounce back if they are forced by the user. For these particles, specialised channels are embedded into the cell membrane and used for transporting the particles. GLUT, the glucose transporter, as well as sodium/potassium channels are included into the virtual environment. Finally, the red and blue cubes in the virtual environment are the haptic cursors which follow the movement of the user's fingers.

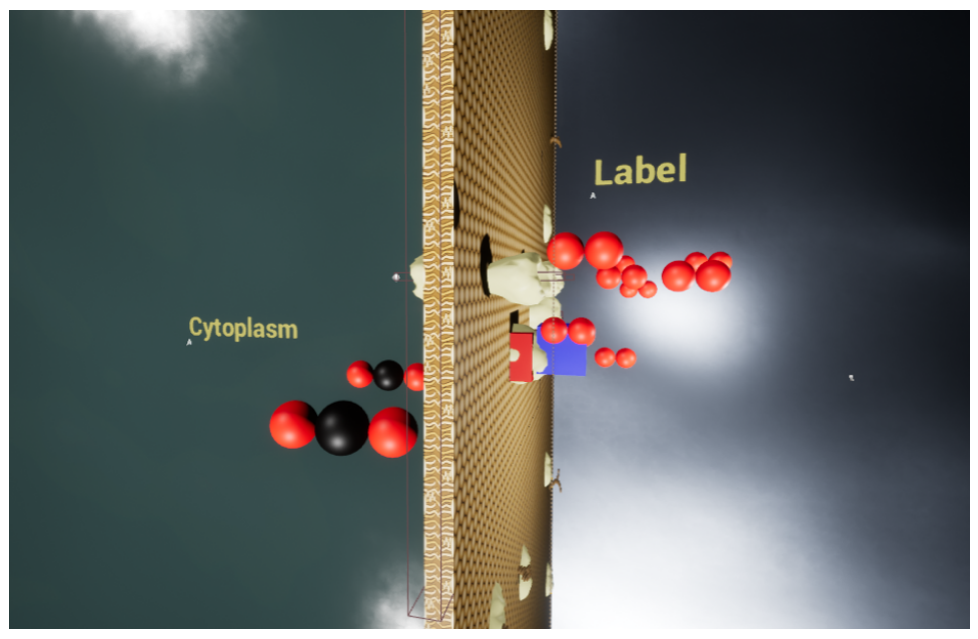

Fig. 2. The virtual environment used in the experiments. The cell membrane divides the world into two halves where the left hand side is the interior of the cell (cytoplasm) and the right hand side is the outside of the cell (extracellular fluid). Oxygen and carbon dioxide molecules are depicted in the figure. Membrane proteins are shown "floating" in the cell membrane. The "Label" shown in the figure is a place holder where the name of the particle grabbed by the user.

The virtual environment was created using Unreal Engine with a Toia add-on developed by Generic Robotics (http://www.genericrobotics.com/). Toia is a haptic add-on that bridges the gap between the graphical capabilities of Unreal Engine and haptic interfaces. It includes drivers for a range of haptic devices including Phantom, Falcon, Omega, W5D. Toia includes a variety of haptic rendering algorithms, including the extended friction cone algorithm for multi-finger haptic rendering. The develop- 
ment environment enables fast prototyping and high quality graphics with high performance graphical rendering.

\section{Experimental Procedure}

The broad aims of this research are to investigate the impact that virtual environments with haptics have in the classroom and to understand their effectiveness in supporting students' learning. As justified in Section 1, this study focuses on biology curriculum, more specifically the concept of diffusion which is taught to Year 8 students.

The preliminary results which is reporting students' responses to interacting with the haptic system (e.g. ease of use, areas for improvement), as this is an important aspect of likely uptake and hence the system's potential to impact learning are presented in this paper. This section describes the procedure for the research study in full, although the following Results section presents findings just from a subset of the data, specifically interviews with the students about their experiences of using the system.

The study consists of three parts. In the first part, the student was given a biology and three psychometric tests. This biology test (pre-test), consists of questions on fundamental biology knowledge and diffusion. The aim of the pre-test is to measure the student's knowledge and reveal misconceptions relating to diffusion; moreover the results of the test are used to measure the learning of the student after the second part of the study which is the hands-on session with the haptic interface.

In addition to the biology pre-test, the student is given 3 psychometric tests: block design test [6], fine dexterity test [1] and spatial reasoning test [22]. The block design test requires the subject to match the pattern of the blocks to the reference patter by aligning the correct faces of 912 blocks. The aim of this test is to measure the spatial visualisation and motor skills of the student. For the fine dexterity test, student uses his/her fingers to put washers on pegs in a fixed amount of time. This test measures the manipulative dexterity of the student. Finally, in the spatial reasoning test, the student is given the view of a three dimensional object from different angles and asked to select the correct shape/geometry from the given shapes/objects. This test measures the spatial understanding of the student.

In the second part, two students form a pair and work on the virtual environment using the haptic interface described in Section 4.1. Instructions about the task and the questions to be answered are given in a worksheet, and students are asked to work through the activity as independently as possible. This format was intended to be similar to how students would typically carry-out experiments in their biology classes. During this part of the study, students interactions with the system and with each other are recorded on video and their voices are additionally captured by a dictaphone. Moreover, the researchers observe each pair and take notes on the interaction of the students with each other, the haptic system and the virtual environment.

In third and the final part of the study, the student is given another biology test, which will be referred to as the post-test and the questions 
are on fundamental biology knowledge and diffusion. After the post test, each pair were interviewed about their experiences with the haptic interface, exploring which aspects they particularly liked, found easy or difficult, which aspects they would change, and ease of collaborating as a pair using the system. The interviews also probed what the students had learned about cell biology during the activity, and how working collaboratively in a pair supported their learning. The interviews are audio recorded.

62 Year 8 students (typically 12-13 years old), 34 boys and 28 girls, participated in the study. Students had experience of conducting traditional biology experiments as a part of their curriculum, however none of the participants had used haptic interfaces before nor had experience of performing virtual experiments on cells. A minority of students had familiarity with head-mounted displays and indicated that they had previously tried the technology.

The study was reviewed according to the research ethics procedures of King's College London, and was given a favourable ethical opinion for conduct. The parents of each student provided written informed consent, including explicit consent for audio and video recording, and each student also provided written assent .

\section{Results}

The interviews were transcribed, and the parts of the interviews that related to students' interactions with the haptic system were examined for key points.

\subsection{Which aspects of interaction did students find easy?}

Students reported that they found the system easy to use and easy to control. Comments on ease of use referred to the usability of the haptic interface, noting that the multi-finger haptic interface in this study allows effortless manipulation of the objects in the virtual environment. On the other hand, comments relating to ease of control referred to the user interface designed for the co-pilot. Students mentioned that application controls such as adding more particles, slowing down and freezing particle motion was easily done with the keyboard short-cuts.

\subsection{Which aspects of interaction did students find difficult?}

The majority of the students mentioned that the size of the objects in the virtual environment was crucial for manipulation and commented that small objects, like $\mathrm{O}_{2}$, were not easy to to grab especially if the object was dynamic.

Some students noted some discomfort with the thimble design mentioned in Section 4.1. They indicated that the thimble would not be very comfortable for use over a longer time period, and also that putting on the thimble, attaching the finger to the haptic robot, should be possible without a help from another person. 


\subsection{What would students change about the VR and haptic system?}

The majority of students stressed two points: a virtual environment which they can walk around and a haptic interface which has greater workspace. These responses reflect students' desire for greater immersion in the virtual environment, without physical (or mechanical) limitations. The cursor representation was another important point mentioned by the students. Instead of using red and blue cubes, one pair suggested using 3D finger models in the virtual environment, so that the system would look more realistic: "I think if I was being really pernickety, I would probably go, instead of having like squares for the fingers, I'd maybe have something more finger like, so it was easier to sort of accept that those are your fingers."

Manipulation with two hands and using more fingers for manipulation were also frequently mentioned in the interviews. One pair mentioned that a glove-like haptic interface might be better-suited for these kind of applications.

\subsection{How easy was it to work collaboratively?}

Students mentioned that they found it easy to collaborate using the haptic application. They noted that sharing the same view with the pilot and having the control of the keyboard for interacting with the virtual environment eased collaboration for the co-pilot.

\subsection{Were there any barriers to working together effectively?}

Majority of the students argued that the head-mounted display that the pilot was wearing isolated him/her from the environment; therefore, the communication between the pilot and the co-pilot was harder than faceto-face communication. This is illustrated by the following excerpt of an exchange between the interviewer (IV), and two students (SP1 and SP2):

IV Okay. Anything else you found easy?

SP2 Yes, probably being a co-pilot is much more less stressful.

IV Oh really.

SP2 Yes.

IV Okay. But you do agree with that SP1 ?

SP1 Well, it's quite hard to communicate what you want the guy

to do, and when you're co-pilot he's got the headset on.

On the other hand, from the co-pilot's point of view, not being able to point out an object to the pilot, who is wearing the head-mounted display, was considered as a disadvantage in terms of collaboration. 


\section{Discussion}

Haptic interfaces have been shown to be helpful for learning new skills such as training surgeons. However, using a haptic interface in the classroom for supporting the learning of science subjects has not been explored as extensively. In this work, using biology education as the background, we tested a haptic application and observed how it was used by the students.

We have observed that a haptic application for enhancing learning in a classroom has to be designed very carefully. The important parameters for a successful haptic application in the classroom are the mechanical design of the haptic interface, the usability of the user interface, usability of the graphical display components, and the design of the virtual environment.

The mechanical design of the haptic interface is an important parameter for a successful haptic application since it enables the student to discover the environment so that the underlying learning objectives are met. The multi-finger haptic interface used in this study is a competent example for haptic interfaces for classroom environments since it is intuitive to use. The device acts as an extension of the human fingers to the virtual world and requires little cognitive load for manipulation. Getting used to manipulation with the multi-finger haptic interface is easy and we observed that almost all students had no difficulties using the device even on their first use.

For a system like the multi-finger haptic interface, attaching the user to the haptic system is very crucial. A simple linkage design like the thimble that was used in this study, can undermine the haptic experience. Achieving the ultimate design which fits all fingers is challenging. The thimble design used in this study uses a Velcro strap to fix the user's finger to the thimble linkage. Even though the Velcro strap provides flexibility to different finger sizes, it was observed during the trials, and understood from the student interviews, that the robustness of the thimble attachment should be improved.

Usability of the user interface, including the keyboard interactions in this study, is very important for productive collaboration between the pilot and the co-pilot. Especially for situations where the pilot is isolated from the environment due to wearing the head-mounted display, the user interface helps the co-pilot to manipulate and control the virtual environment and hence helps the pilot to complete the given task.

Students found the head-mounted display awe-inspiring. However, it was observed and also noted by the students that wearing the head-mounted display is isolating the pilot from his/her environment. The isolation may be advantageous depending on the application; however, for enhancing learning in a classroom environment and promoting collaboration between students, isolation could potentially be considered as a disadvantage. Therefore, when designing a haptic application with a headmounted display, researchers have to be attentive on using this powerful graphical display device. The immersion of the user in the virtual environment is readily apparent; however, the classroom environment favours learning over immersion. 


\section{Conclusion}

In this paper, we present observations and feedback from students from a classroom deployment of a haptic system for supporting biology education. We observed that the haptic systems, combined with VR technology, were well-received and easily used by the students, and these results lend support for the further development and investigation of these systems in science education. Our ongoing work includes measuring the impact of the haptic interaction on students' learning of the scientific concepts and support the preliminary results with thorough quantitative analysis.

Acknowledgements The authors thank the participants for their valuable feedback and are pleased to acknowledge support for this work from The Leverhulme Trust for the project '3D Learning in a Rich, Cooperative Haptic Environment'. We are also pleased to thank our colleagues on this project Jon Rashid, Carleen Houbart, Phil James, Richard Fisher, Simon Bliss, Peter Tolson and Balazs Janko.

\section{References}

1. The morrisby fine dexterity test. http://www.morrisby.com/pages/public/dexterity-tests.aspx, accessed: 29.01.2018

2. Inside a cell (2016), http://learn.genetics.utah.edu/content/ cells/insideacell/

3. Bamford, A.: Evaluation of innovation in learning using emerging technologies. Tech. rep., Gaia Technologies (2011), http: //www.gaia3d.co.uk/wp-content/uploads/2012/11/Evaluationof-Innovation-in-Learning-using-emerging-technologies-byProf-Anne-Bamford-2011.pdf

4. Bolinsky, D.: Visualizing the wonder of a living cell (March 2007), http://www.ted.com/talks/david_bolinsky_animates_a_cell

5. Bowen, K., O'Malley, M.K.: Adaptation of haptic interfaces for a labview-based system dynamics course. In: Haptic Interfaces for Virtual Environment and Teleoperator Systems, 2006 14th Symposium on. pp. 147-152. IEEE (2006)

6. D, W.: Intelligence Scale for Children. NCS Pearson (2014)

7. Ernst, M., Banks, M.: Humans integrate visual and haptic information in a statistically optimal fashion. Nature 415(6870), 429-433 (2002)

8. Gauldie, D., Wright, M., Shillito, A.: 3D modelling is not for WIMPS part ii: Stylus/mouse clicks. In: Proceedings of Eurohaptics. pp. 182189 (2004)

9. Gillespie, R., Hoffinan, M., Freudenberg, J.: Haptic interface for hands-on instruction in system dynamics and embedded control. In: Haptic Interfaces for Virtual Environment and Teleoperator Systems, 2003. HAPTICS 2003. Proceedings. 11th Symposium on. pp. 410-415 (March 2003) 
10. Johnson, R.: 3D at The Abbey. Engineering \& Technology Education pp. 12-15 (June 2011)

11. Macaulay, K.: Use of 3d technologies in the classroom (April 2012), http://www.3deducationconference.org/, international Education and Technology Conference

12. Melder, N., Harwin, W.: Extending the friction cone algorithm for arbitrary polygon based haptic objects. In: Haptic Interfaces for Virtual Environment and Teleoperator Systems, 2004. pp. 234 - 241. IEEE (2004), http://ieeexplore.iee.org/ie15/9655/ 30512/01407005.pdf

13. Millar, R.: Practical work. In: Osborne, J., Dillon, J. (eds.) Good Practice In Science Teaching: What Research Has To Say, pp. 108134. Open University Press, Maidenhead, second edn. (2010)

14. Okamura, A., Basdogan, C., Baillie, S., Harwin, W.: Haptics in medicine and clinical skill acquisition. IEEE Transactions on Haptics 4(3), 153-154 (2011)

15. Okamura, A.M., Richard, C., Cutkosky, M., et al.: Feeling is believing: Using a force-feedback joystick to teach dynamic systems. Journal of Engineering Education 91(3), 345-349 (2002)

16. Otaran, A., Tokatli, O., Patoglu, V.: Hands-on learning with a series elastic educational robot. In: Haptics: Perception, Devices, Control, and Applications (EuroHaptics). pp. 3-16 (2016)

17. Piburn, M.D., Reynolds, S.J., McAuliffe, C., Leedy, D.E., Birk, J.P., Johnson, J.K.: The role of visualization in learning from computerbased images. International Journal of Science Education 27(5), 513527 (2005)

18. Rose, C.G., French, J., O'Malley, M.K., et al.: Design and characterization of a haptic paddle for dynamics education. In: Haptics Symposium (HAPTICS), 2014 IEEE. pp. 265-270. IEEE (2014)

19. Rutten, N., van Joolingen, W.R., van der Veen, J.T.: The learning effects of computer simulations in science education. Computers \& Education 58(1), 136-153 (2012)

20. Tse, B., Harwin, W., Barrow, A., Quinn, B., Diego, J.S., Cox, M.: Design and development of a haptic dental training system haptel. In: Lecture Notes in Computer Science. vol. 6192 Part II, pp. 101-108 (2010), http://www. springerlink. com/content/9783-642-14074-7\#section=726919\&page $=1$

21. Tuckey, H., Selvaratnam, M.: Studies involving three-dimensional visualisation skills in chemistry: A review. Studies in Science Education 21(1) (1993)

22. U, L.J., N, L.: Mechanical Aptitude and Spatial Relations Tests. Thomson Learning (1999)

23. Webb, M.: Impact of it on science education. In: International handbook of information technology in primary and secondary education, pp. 133-148. Springer (2008) 